\title{
ROLE OF HUMAN PAPILLOMAVIRUS IN CARCINOGENESIS OF DIGESTIVE TRACT
}

\author{
${ }^{1,2}$ Reva G.V., ${ }^{1,2}$ Tolmachev V.E., ${ }^{1,3}$ Dynda Yu.V., ${ }^{1,2}$ Kim A.R. \\ ${ }^{1}$ International Medical Education and Research Center, Vladivostok, e-mail: revagal@yandex.ru; \\ ${ }^{2}$ Far-Eastern State University, Vladivostok, e-mail: tolmachev-ve@mail.ru; \\ ${ }^{3}$ Pacific State Medical University, Vladivostok, e-mail: dyndaiurii@gmail.com \\ High risk human papillomavirus infection is the most common sexually transmitted infection due to the latest \\ report of the WHO. About $90 \%$ of all cervical cancers are caused by human papillomavirus, meanwhile about $30 \%$ \\ of oropharyngeal carcinoma's new cases are presented with HPV-positive status, and its percentage's still growing. \\ The abovementioned facts indicate that in the modern world there is a need for more detailed study on the issue \\ related to the carcinogenic effect of human papillomavirus. This article focuses primarily on the main issues of HPV \\ carcinogenic effect in the mucous membrane of oropharynx, revealing the main characteristic features of immuno- \\ logical landscape, gene expression profile and its significance in the context of 5-years overall survival. These data \\ explains the importance of cellular and humoral immunity in the development of the proinflammatory antitumor \\ environment and suggests information about the influence of the gene expression levels for the long-term prognosis \\ of patients diagnosed with pathology of HPV-positive oropharyngeal squamous cell carcinomas.
}

Keywords: high risk human papillomavirus, oropharyngeal squamous cell carcinoma, gene expression profile, T cells, myeloid cells, cytokines, IFI16, MHC-II

Contrary to the popular belief of human papillomavirus to be predominantly a lot of marginal segments of population, current research materials have established the opposite. In accordance with the latest statistical reports, more than $12 \%$ of the World's population are the carriers of papillomavirus besides more than $3 \%$ suffer from HPV-associated cancer pathologies, irrespective of one's gender and age, socio-economic status and ethnicity.

The dominant role of human papillomavirus in cervical cancer is generally recognized and well-known scientific fact, but over the past thirty years, the scientific community has accumulated sufficient information on the subject of HPV's carcinogenic role in alternative anatomical locations.

Due to the statistical data obtained by the European Institute of Oncology (EIO) for the period of 2000-2010, out of 675 patients with confirmed HPV-positive status, $48.1 \%$ of patients were diagnosed with cancer of the oral cavity, pharynx and oropharyngeal region $(1.8 \%, 2.2 \%, 40.4 \%$, respectively) [1]. In accordance with other scholars, the incidence of HPV-positive oropharyngeal cancer in the period of 1988-2004 was increased by $225 \%$ and at the present moment constitutes about $90 \%$ of all cases of oropharyngeal cancer among American population, and pursuant to $\mathrm{Ca}$ nadian research centers, the frequency of the disease was increased from $40 \%$ up to $74 \%$ during the period of 2000-2012 [2]. Therefore we have strong statistical evidence that incidence of $\mathrm{HPV}^{+}$oropharyngeal squamous cell carcinoma has been increasing dramatically. Predisposing factors towards HPV-positive OPSCC are alcohol abuse, tobacco smoking and promiscuity, meanwhile the lack of timely diagnostics leads to the problem of oropharyngeal cancer to be most frequently detected at advanced stages.

This article presents the main current information on the pathological features of high-risk human papillomavirus-induced oropharyngeal squamous cell carcinoma.

\section{Purpose of the study}

To study the most informative diagnostic resource of HPV-induced oropharyngeal squamous cell carcinoma in oncological practice.

\section{Materials and research methods}

The study was carried out in accordance with the Declaration of Helsinki 2000-2013, with the permission of the Ethics Committee of the FGAOU HE FEFU. The preparations made from biopsies of the oral mucosa of 7 patients were studied according to clinical indications. The biopsy material was obtained in accordance with the order of the Ministry of Healthcare of the Russian Federation dated 04.29.94 N 82 "On the procedure for taking biopsy material", according to the rules of the instructions governing the procedure for laboratory tests in medical institutions. The control group consisted of 3 patients who died as a result of injuries incompatible with life, presumably without somatic pathology at the age from 34 to 46 years. The biopsy material was fixed according to the prescription for preparation for histological examination immediately after collection, and then the sections were stained with hematoxylin and eosin. The presence of HPVI in patients with COP papillomas was confirmed by PCR. The method of immunohistochemistry to identify the localization of cells expressing Ki67 and P63 was performed 
according to the standard DAKO protocol. The results were analyzed using the standard variation statistics method. The analysis of the preparations was performed using an Olympus microscope with proprietary software. The analysis of the available literature data on the inductive role of human papillomavirus in the carcinogenesis of squamous cell carcinoma of the mucous membranes of the gastrointestinal tract was performed.

\section{Research results and discussion}

Human papillomavirus consists of two main groups of proteins presented by Early proteins (E1, E2, E3, E4, E5, E6, E7, E8) and Late proteins (L1 and L2). The mentioned proteins are involved in the metabolism of the epithelial cells of the mucous membrane affected by the papillomavirus as it starts to function in the host-cell.

However, the leading role in carcinogenesis is played by proteins E6 and E7, which is why they are called oncoproteins. They lead to irreversible changes in the histological picture of the tissue, which is expressed in the form of abnormal epithelial proliferation and the development of malignant neoplasia of the mucous membrane. In modern oncological practice, doctors often encounter papillomavirus-induced malignancies, and one of such HPV-induced malignant pathologies is oropharyngeal squamous cell carcinoma.

The evidence of different pathogenesis and prognosis of squamous cell carcinoma of the oropharyngeal region depending on HPV-status has been repeatedly urged in modern scientific literature. This difference is primarily based on distinct immunological characteristics of tumors and notable gene expression patterns of their cells, depending on the presence of papillomavirus infection.

There are a number of publications claiming the HPV-induced oropharyngeal cancer to be much easier to control and to have substantially more promising prognosis in the context of five-year patient's survival. Such feature is explained by the presence of a more functionally active immunocompetent cellular infiltrate, more active accumulation of humoral immunity substances around cancer cells, and special pattern of gene expression profile in the pathological cells themselves.

R. Hewavisenti, A. Ferguson et al. (2020), examining squamous cell carcinoma tissues with HPV-positive status, noted a high level of intratumoral and stromal infiltration by $\mathrm{CD} 103^{+} \mathrm{CD}^{+} \mathrm{T}$ cells, which was associated with an improved outcome of HPV-positive cancer compared to HPV-negative $(83.6 \%$ and $53.9 \%$, respectively), while CD103-CD ${ }^{+}$ $\mathrm{T}$ cells were evenly distributed through the stroma of $\mathrm{HPV}^{+/}$tumors and had no prognostic significance [3].

Other researchers (S.J Santegoets, Ch.L Duurland et al.(2020)) determined a strong cellular infiltration of the tumor by $\mathrm{CD} 14^{+} \mathrm{CD} 33^{-\mathrm{CD}} 163^{+}$, $\mathrm{CD}_{14}{ }^{+} \mathrm{CD} 33^{+} \mathrm{CD} 163^{+}$and $\mathrm{CD} 14-\mathrm{CD} 33-\mathrm{CD} 163^{+}$ cells, and, in particular, an increased content of CD14-CD33 ${ }^{+} \mathrm{CD} 163^{-}$immature myeloid cells and CD14-CD33-CD163+ cells in the tumor stroma was noted. Thereafter, by studying the correlation between the total number of $\mathrm{CD}^{+}$cells and CD14-CD33-CD163 ${ }^{+}$myeloid cells, CD8 $^{+}$Foxp3Tbet $^{+}$and CD8-Foxp3-Tbet ${ }^{+}$(CD4) cells, the accumulation of CD14-CD33-CD163+ ${ }^{+}$around tumor cells was found to provide a cancer-specific immune response and, consequently, a positive long-term prognosis.

In another part of the study, the same authors identified the combined stimulating effect of CD14-CD163- and CD14-CD163 ${ }^{+}$dendritic cells on the proliferation of the T-cellular population and production of first-type cytokines (INF $\gamma$, IL-22), while CD14-CD163- dendritic cells also had the ability to stimulate the production of cytokines of the second type (IL-9, IL-13). It has been experimentally proven that CD $144^{-} \mathrm{CD} 163^{+}$ cells are able to secrete a large number of proinflammatory mediators (IL-12p70, IL-18, IL-10, IL-23, IL-6, IL-1 $\alpha$, IL-1 $\beta$, MIP1 $\alpha$, MIP3 $\alpha$, TGF $\alpha$, $\mathrm{TNF} \alpha$ ) along with chemokines and cytokines responsible for chemoattraction and activation of immunocompetent cells (IL-1 $\beta$, IL-15, IL-16, IL-18). Notably, CD14-CD163 ${ }^{+}$expressed the TLR3, CXCR2, and CXCR4 genes more strongly than $\mathrm{CD} 14^{+} \mathrm{CD} 163^{+}[4]$.

P. Baruah, J. Bullenkamp et al. (2019) conducted the study, which confirmed the presence of PD-L1 and PD-L2 protein expression during pathogenesis of papillomavirus-induced squamous cell carcinoma. These proteins were expressed on fibroblasts and macrophage cells. Subsequently, it was experimentally established that the regulation of PD-L1 and PD-L2 protein expression is controlled directly by affecting TLR9-receptors. The processes in question ensure the presence of the certain microenvironment, predisposing to the development of tumor pathology in the tissue, since it is PD-1 that has the ability to inhibit the activity of T-lymphocytes and, as a result, prevent the development of a robust antitumor response of the body [5].

D. Mytilineos, A. von Witzleben et al. (2020), who studied the peripheral level of cytokines during papillomavirus-positive oropharyngeal squamous cell carcinoma, found a high plasma content of proapoptotic immune 
mediators (Granzymes A and B, cytokines IFN $\gamma$, GMCSF, sFasL, TNF $\alpha$, sCD137, IL-2, IL-4, IL-5, IL-6, IL-10, IL-13, HMGB1), among which sFas-mediators and perforins were the most prevalent ones. Perforins integrated into the cell membrane form pores through which granzymes are transported to the target cells and thereby cytotoxic $\mathrm{T}$ cell and NK cell response is provided. Based on the presented data, the authors claim a robust inflammatory phenotype to be present in patients with $\mathrm{HPV}^{+}$cancers of oropharynx, and, in this regard, it is concluded that the course of virusinduced squamous cell oropharyngeal cancer has much more favorable prognosis [6].

At the same time, M.J.P. Welters et al. (2020), considering the question of immunological characteristics of HPV+ OPSCC, emphasize the correlation between CD8+ and CD4+ T cells tumor infiltrations and a longterm favorable prognosis. The authors' material provides information on the subject of an increased level of IFN $\gamma$ and a reduced content of IL- 4 and TGF $\beta$ in tumor tissue produced by CD8+ T cells [7].

The studies presented by F.O. Gleber-Netto, H.D. Skinner et al. (2019) indicate that there are some genes, which possess certain predictive significance due to their influence on the pathogenesis of the disease. Among them, IKZF3, ARHGAP26, and CACNA1D are marked as the genes with the highest expression level. The gene of IKZF3 is known to be a lymphocytic transcriptional factor which regulates cellular processes of apoptosis and differentiation, another representative of the cohort CACNA1D is a regulator of calcium $\mathrm{CaV} 1.3$ channels, and the last one ARHGAP26 is a gene encoding the Rho GTP-ase protein activation [8].

Altogether, the influence of the factors should lead to the course of cancer tissue metabolism according to the stable scenario. In addition to the abovementioned material, more elaborate information obtained by $R$. Alex Harrison et al. (2018) presents the fact of a direct relationship between somatic mutations in the genes of tumor cells and the probability of recurrence of the pathology. Epigenetic regulation genes (KMT2D), Notch-Signaling Pathway Regulation genes (SPEN), Cell Motility genes (ANK3), Mitogenetic Signaling genes (FGFR3), and the genes of Cellular Differentiation (ZNF750), as well as FGFR3, PTEN, PIK3R1, CYLD were noted as the most susceptible to mutational factor among the total number of all cancer cases, and, in their turn, FTL1, PIK3R6, TSC2, KMT2D, MTOR, ALK, NFE2L2, and FGFR3 were the most suscep- tible to mutations among the cases of cancer recurrence in particular [9].

According to our data, with HPV-positive status in the oral mucosa, there is an increase in the proliferative activity of keratinocytes, apoptosis in the basal layers and a decrease in the processes of differentiation of the epithelium, which reduces its barrier properties (Figure).

More detailed information, regarding the role of CXCL14 in antigen-specific $\mathrm{CD}^{+} \mathrm{T}$ cell activity, was received by American scientists J.A. Westrich, D.W. Vermeer et al. (2019) Activation of the gene leads to suppression of tumor cell growth, restoration of MHC-I H-2db on the surface of HPV-infected tumor cells, and better intratumoral infiltration by $\mathrm{CD}^{+} \mathrm{T}$ cells [10].

Investigating the role of $\mathrm{MHC}-\mathrm{II}$ during papillomavirus-induced squamous cell carcinoma, S.F. Gameiro, F. Ghasemi et al. (2019) revealed the data of strong expression of HLA-DPA1, -DPB1, -DQA1, -DQA2, -DQB1, -DQB2, -DRA, -DRB1, -DRB5, -DRB6 genes of the $\alpha$ and $\beta$ chains of the main histocompatibility complex of the second type altogether with a significantly increased level of TNFRSF4, CD132 (and its ligand TNFSF4, CD252) and CD74, the last of which responsible for the antigen presentation [11].

Pursuant to M.A. Prusinkiewicz, S.F. Gameiro et al. (2020), the genes involved in the process of glycolysis were down-regulated in the tumor tissue; meanwhile the genes involved in the oxygen phosphorylation, $\beta$-oxidation and tricarboxylic acid cycle were distinctively upregulated. Besides, a number of genes - SDHC, COX7A1, COX16, COX17, ELOVL6, GOT2, and SLC16A2 - directly correlated with a more favorable long-term prognosis [12].

Another notable study conducted by E. Reeves, O. Wood et al.(2019) revealed direct correlation between the level of ERAP1 protein and the amount of $\mathrm{CD}^{+}$lymphocytic tumor infiltrate. The authors noted that the activity of the ERAP1 protein directly controls the recognition of epitopes derived from papillomavirus proteins, which provokes the emergence of robust anti-tumor T cell response [13].

In turn, G. Rivo, M. Biolatti et al.(2020), defined the role of PYHIN proteins, which perform the function of DNA sensors in the body. Of all PYHIN proteins, the most interesting in the context of the study was IFI16, the protein responsible for the processes of apoptosis, senescence, and cellular response to DNA damage, differentiation, and cell growth. According to the authors' idea, the protein is of the key importance during the processes of limitation viral activity inside the infected cancer cells [14]. 

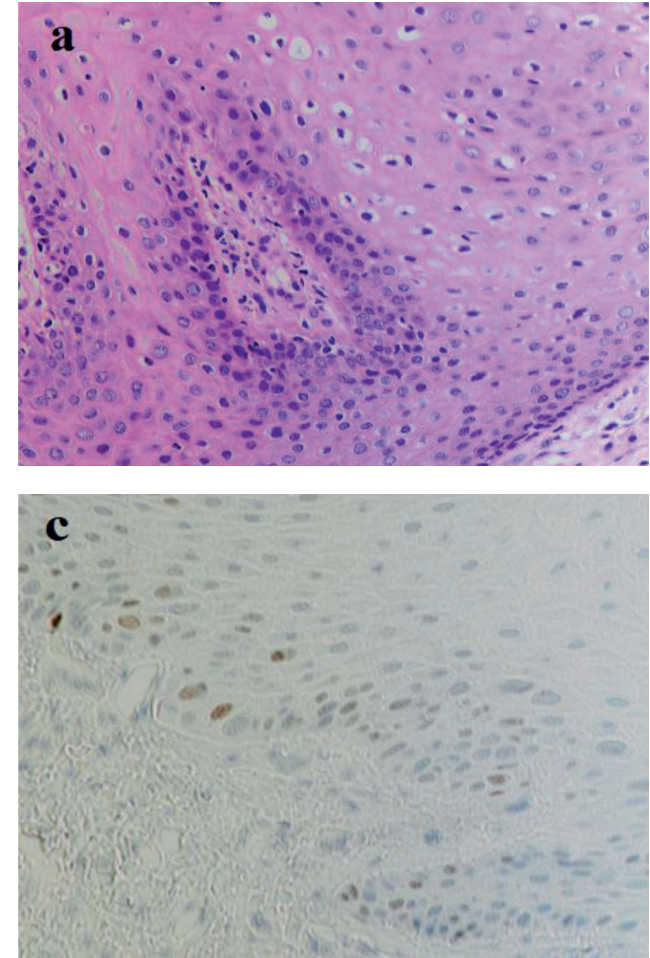
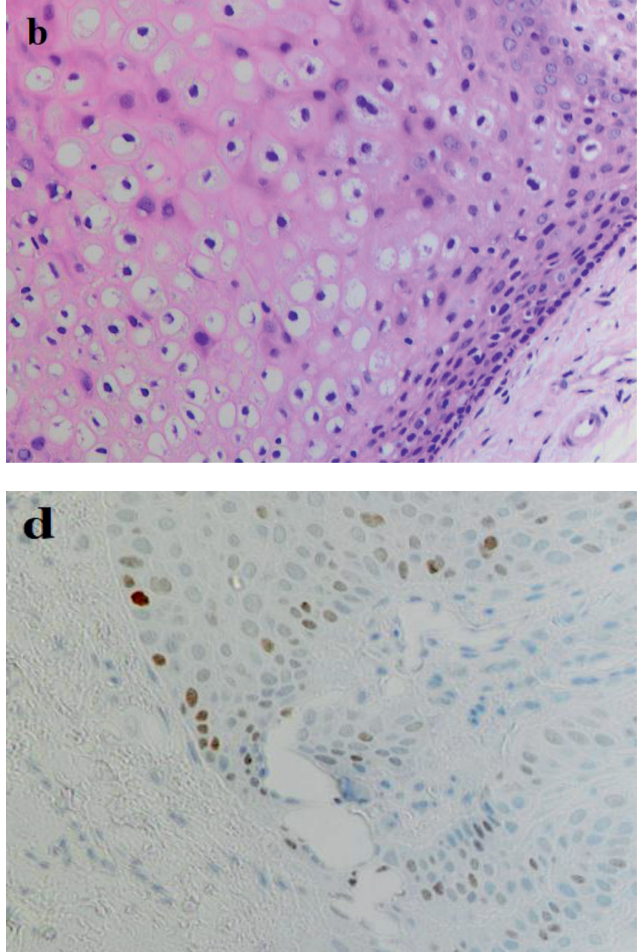

COP of a patient with HPV-positive status. (A, B) Staining with hematoxylin and eosin; (C, D) Immunohistochemistry to identify the localization of cells expressing P63. Microphoto. X 200

\section{Conclusion}

Summarizing the abovementioned scientific information, one is forced to conclude that in fact, papillomavirus infection has a powerful carcinogenic potential influence on epithelial cells of mucosa membrane and remains to be the problem of the upmost importance in the modern oncological practice. Unfortunately, the latest statistical data indicates a rapid increasing in frequency of HPV-associated malignancies. Therefore a further study of pathogenetic mechanisms of papillomavirus infection is a key task in finding new strategies of prevention and treatment HPV-positive squamous cell carcinoma of the oropharyngeal region.

\section{References}

1. Tagliabue M., Mena M., Maffini F., Gheit T., Quirós Blasco B., Holzinger D., Tous S., Scel-si D., Riva D, Grosso E., Chu F., Lucas E., Ridder R., Rrehm S., Bogers J.P., Lepanto D., Lloveras Rubio B., Vijay Kumar R., Gangane N., Clavero O., Pawlita M., Anantharaman D., Radhakrishna Pillai M., Brennan P., Sankaranarayanan R., Arbyn M., Lombardi F., Taberna M., Gandini S., Chiesa F., Ansarin M., Alemany L., Tommasino M., Chiocca S., The HPV-AHEAD Study Group. Role of Human Papillomavirus Infection in Head and Neck Cancer in Ita-ly: The HPV-AHEAD Study. Cancers 2020, 12, 3567. DOI: 10.3390/cancers 12123567.

2. You E.L., Henry M., Zeitouni A.G. Human papillomavirus-associated oropharyngeal cancer: review of current evidence and management. Curr Oncol. 2019 Apr;26(2):119-123. DOI: 10.3747/co.26.4819.
3. Hewavisenti R., Ferguson A., Wang K., et al. CD103+ tumor-resident $\mathrm{CD} 8+\mathrm{T}$ cell numbers under-lie improved patient survival in oropharyngeal squamous cell carcinoma. Journal for ImmunoTherapy of Cancer 2020;8:e000452. DOI: 10.1136/ jitc-2019-00045.

4. Santegoets S.J., Duurland C.L., Jordanova E.J., et al. CD163+ cytokine- producing cDC2 stimulate intratumoral type $1 \mathrm{~T}$ cell responses in HPV16-induced oropharyngeal cancer. Journal for ImmunoTherapy of Cancer 2020;8:e001053. DOI: 10.1136/ jitc-2020-001053.

5. Baruah P., Bullenkamp J., Wilson P.O.G., Lee M., Kaski J.C., Dumitriu I.E. (2019) TLR9 Mediated Tumor-Stroma Interactions in Human Papilloma Virus (HPV)-Positive Head and Neck Squamous Cell Carcinoma Up-Regulate PD-L1 and PD-L2. Front. Immunol. 2019 10:1644. DOI: 10.3389/fimmu.2019.0164.

6. Mytilineos D., Ezić J., von Witzleben A., Mytilineos J., Lotfi R., Fürst D., Tsamadou C., Theodoraki M.N., Oster A., Völkel G., Kestler H.A., Brunner C., Schuler P.J., Doescher J., Hoffmann T.K., Laban S. Peripheral Cytokine Levels Differ by HPV Status and Change Treatment-Dependently in Patients with Head and Neck Squamous Cell Carcinoma. Int J Mol Sci. 2020 Aug 20;21(17):5990. doi: 10.3390/ijms21175990. PMID: 32825343; PMCID: PMC7503943.

7. Welters M.J.P., Santegoets S.J. and van der Burg SH (2020) The Tumor Microenvironment and Im-munotherapy of Oropharyngeal Squamous Cell Carcinoma. Front. Oncol. 10:545385. DOI: $10.3389 /$ fonc. 2020.545385 .

8. Gleber-Netto F.O., Rao X., Guo T., Xi Y., Gao M., Shen L., Erikson K., Kalu N.N., Ren S., Xu G., et al. Variations in HPV function are associated with survival in squamous cell carcinoma. JCI Insight. 2019;4(1):e124762. DOI: 10.1172/jci.insight.124762.

9. Harrison R.A., Duvuri U., et al. The mutational landscape of recurrent versus nonrecurrent human papillomavirusrelated oropharyngeal cancer. JCI Insight. 2018;3(14):e99327. DOI: 10.1172 jeci.insight.99327.

10. Westrich J.A., Vermeer D.W., Silva A., Bonney S., Berger J.N., Cicchini L., et al. CXCL14 supresses human papil- 
lomavirus-associated head and neck cancer through antigen specific CD8+ T cell responses by upregulating MHC-I expression. Oncogene. 2019 November ; 38(46): 7166-7180. doi:10.1038/ s41388-019-0911-6.

11. Gameiro S.F., Ghasemi F., Barrett J.W., Nichols A.C., Mymryk J.S. High Level Expression of MHC-II in HPV+ Head and Neck Cancers Suggests that Tumor Epithelial Cells Serve an Important Role as Accessory Antigen Presenting Cells. Cancers 2019, 11, 1129. DOI: 10.3390/cancers11081129.

12. Prusinkiewicz M.A., Gameiro S.F., Ghasemi F., Dodge M.J., Zeng P.Y.F., Maekebay H., Barrett J.W., Nichols A.C., Mymryk J.S. Survival-Associated Metabolic Genes in
Human Pap-illomavirus-Positive Head and Neck Cancers. Cancers 2020, 12, 253. DOI: 10.3390/cancers 12010253 .

13. Reeves E.,Wood O., Ottensmeier C.H.,King E.V., Thomas G.J., Elliott T. and James E. HPV Epitope Processing Differences Correlate with ERAP1 Allotype and Extent of CD8+ T-cell Tumor Infiltration in OPSCC. Cancer Immunol Res July 12019 (7) (7) 1202-1213. DOI: 10.1158/2326-6066. CIR-18-0498.

14. Giuseppe Riva, Matteo Biolatti, Giancarlo Pecorari, Valentina Dell'Oste, Santo Landolfo Micro-organisms. 2020 Jan; 8(1): 14. Published online 2019 Dec 20. DOI: 10.3390/ microorganisms 8010014 . 